\title{
Diversificação e Desempenho em Empresas Industriais Brasileiras: um Estudo Empírico no Período de 1997 a 2001
}

\section{Effects of the Diversification Strategies on the Performance of the Brazilian Industrial Companies: a Study about Companies Listed in the São Paulo Stock Exchange in the Period 1997-2001}

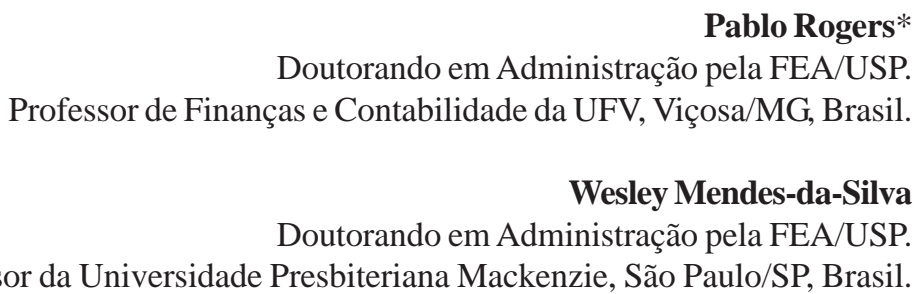

Professor da Universidade Presbiteriana Mackenzie, São Paulo/SP, Brasil.

Germano Mendes de Paula

Pós-Doutorado em Economia pela University of Oxford, Reino Unido. Professor do Instituto de Economia da UFU, Uberlândia/MG, Brasil.

*Endereço: Rua Presidente Médici, 128, apto. 102 B, Clélia Bernardes, Viçosa/MG, 36570-000.E-mail:msc_pablo@yahoo.com.br 


\title{
Resumo
}

Diversos estudos, em diferentes países, têm buscado verificar a associação existente entre a diversificação de negócios e o desempenho corporativo. Essencialmente, três modelos têm sido testados para descrever essa associação: o modelo linear, o modelo curvilíneo ('U invertido') e o modelo intermediário. Esse trabalho tem por objetivo estudar esta possível relação a partir de evidências empíricas que dizem respeito às empresas industriais brasileiras. Usando dados de companhias de capital aberto no período $1997-$ 2001, procedeu-se a uma análise de painel por meio dos métodos de Efeitos Fixos e Efeitos Aleatórios. Os dados não apresentaram aderência suficiente para aceitar a existência de um efeito positivo entre estratégias de diversificação e desempenho, seja linearmente seja exponencialmente. Assim, o modelo que apresentou melhor grau de ajuste aos dados foi o modelo curvilíneo por meio das formas funcionais quadrática e cúbica, o que sugere a existência de pontos em que os custos marginais da diversificação superam os benefícios marginais e vice-versa. Porém a relação mostrou-se indefinida, não sendo possível concluir se ocorre sob a forma de ' $U$ ' ou de 'U invertido'. Os principais resultados obtidos na pesquisa apóiam algumas evidências empíricas encontradas em outros países emergentes.

Palavras-chave: estratégias corporativas; diversificação; desempenho financeiro.

\begin{abstract}
Several studies, in different countries, have been investigating the relationship between diversification of businesses and corporate performance. Basically, three models have been tested to describe that relationship: the linear model, the curvilinear model ( 'inverted U'), and the intermediate model. This paper focuses on theme, using the Brazilian industrial companies as the empirical evidence. The study scrutinizes the performance of enterprises that were listed in the São Paulo Stock Exchange in the period 1997-2001, via a panel analysis through the methods of Fixed Effects and Random Effects. The data didn't show enough adherence to accept that a positive relationship exists between diversification strategies and performance, neither lineally nor exponentially. The model that showed a better adjustment degree was the curvilinear model (through the quadratic and cubic functional forms) that can be interpreted as an evidence about the existence of points in which the marginal costs of diversification overcomes the marginal benefits and viceversa. However, the relationship was shown indefinite; it was unable to conclude if the real shape is similar to an ' $U$ ' of an 'inverted U'. The main results obtained in the research support some empirical evidences found in other emerging countries.
\end{abstract}

Key words: corporate strategies; diversification; financial performance. 


\section{INTRODUÇÃO}

A década de 1990 foi um período emblemático no Brasil, em função das intensas mudanças verificadas nos regimes de incentivos e de regulação da atividade econômica. Nesse contexto, a expectativa seria de que as empresas redefinissem suas estratégias corporativas e competitivas para se adequar a um novo ambiente institucional. As estratégias corporativas dizem respeito às estratégias de empresas que já são diversificadas ou que estão entrando em novos negócios (diversificação) ou saindo de algum deles (focalização). Tais estratégias também envolvem o estabelecimento de prioridades entre as unidades de negócios, e ainda o relacionamento entre as subsidiárias e a holding. Em suma, as estratégias corporativas consistem em duas questões principais: i) em que negócios a empresa deve atuar; ii) como o escritório central gerencia cada um de seus negócios (Hitt, Ireland, \& Hoskisson, 2001, p. 232).

A literatura sobre estratégias empresariais é fortemente baseada na experiência de companhias com sede em países desenvolvidos, especialmente nos Estados Unidos. O tema das estratégias corporativas segue esta tendência. Embora pelo menos três correntes possam ser identificadas, a visão predominante é de que a diversificação conglomerada (ou não-relacionada) acarrete impactos negativos sobre o desempenho empresarial e, por conta disto, as empresas deveriam concentrar suas atividades em torno de suas competências essenciais (Moreira \& Planellas, 2003).

Com relação à diversificação de empresas com sede em países em desenvolvimento, Nachum (1999) argumenta que vários mercados domésticos não são suficientemente grandes para permitir o crescimento da empresa em apenas uma indústria. Ademais, algumas economias estão sujeitas a grandes riscos e à instabilidade decorrente da rápida mudança estrutural. Isto estimularia a diversificação como meio de se evitar o risco da especialização em apenas uma indústria. Em muitos países em desenvolvimento existiria a tradição de que cada membro da família tenha uma parte igual na empresa para administrar. E a falta de tecnologia proprietária por parte das empresas implicaria a inexistência de competências a serem exploradas em atividades relacionadas. Pelo contrário, a aquisição externa de tecnologia possibilitaria uma trajetória de crescimento baseado na diversificação conglomerada.

Ademais, nos países em desenvolvimento em geral, e na América Latina em particular, a estrutura de propriedade e o grau de especialização produtiva são bastante distintos dos padrões verificados nos Estados Unidos, por exemplo. $\mathrm{Na}$ 
economia latino-americana, várias grandes empresas privadas domésticas são controladas por famílias, que também se encarregam da gestão, ao mesmo tempo que o grau de diversificação produtiva é maior. Tendo em vista a alta concentração de propriedade, o mercado de controle corporativo é pouco relevante. Ademais, são poucas as empresas latino-americanas que possuem relevantes investimentos diretos em outros países. A intensidade do esforço tecnológico das companhias latino-americanas é relativamente baixa em comparação com o verificado em países desenvolvidos. Por isso o formato empresarial típico de empresas privadas latino-americanas é muito distinto do que se poderia denominar de 'paradigma anglo-saxão'.

Diante das significativas diferenças apontadas entre os ambientes e as configurações empresariais verificadas nos países latino-americanos vis-à-vis da experiência norte-americana, cabe indagar se as motivações e os resultados da diversificação seriam distintos nessas duas realidades. Nesse sentido, esse artigo contribui para o debate recente sobre a relação entre diversificação e desempenho empresarial na América Latina, tomando o Brasil como exemplo. Simultaneamente, investiga-se em que medida os resultados encontrados se aproximam ou se distanciam dos verificados para a experiência norte-americana. O presente texto está composto de cinco seções, incluindo esta Introdução. A segunda seção é dedicada ao resgate das bases teóricas da pesquisa, ao passo que a terceira apresenta a metodologia. Os resultados empíricos obtidos na pesquisa são apresentados e discutidos na seção subseqüente. A quinta seção sumaria as principais conclusões deste artigo.

\section{REVISÃo dA Literatura}

\section{Estratégias de Diversificação em Países Desenvolvidos}

No que tange às estratégias empresariais de diversificação, a visão dominante na literatura encontra-se fortemente baseada na experiência de companhias com sede nos países desenvolvidos de modo geral, e nos Estados Unidos em particular. Observa-se porém que, freqüentemente, estudos realizados na América Latina tomam preferencialmente o paradigma norte-americano como parâmetro de comparação. Verifica-se na experiência norte-americana que, na década de 1920, várias firmas optaram pela integração vertical, enquanto, a partir de meados da década de 1960, as companhias privilegiaram a diversificação produtiva. Estimase que, no âmbito das F\&As realizadas nos Estados Unidos entre 1948 e 1978, cerca de $40 \%$ dos ativos foram direcionados à diversificação relacionada, $34 \%$ à 
diversificação conglomerada, 17\% à horizontalização e 9\% à integração vertical (Karier, 1993, p. 145).

No entanto, a partir do final dos anos 1970 e na primeira metade dos anos 1980 , constatou-se uma drástica reversão da tendência de aumento da relevância da diversificação produtiva, pois as empresas norte-americanas e britânicas começaram retomar a concentração das suas atividades produtivas. Dentre as 500 maiores companhias industriais norte-americanas elencadas pela revista Fortune, em 1950, mais de 60\% delas obtinham mais de $70 \%$ de suas receitas com um negócio. Em 1974, esse percentual já tinha regredido para 37\%, voltando, então para 53\% em 1988 (Hitt et al., 2001, p. 233). A Figura 1 ratifica que, a partir de meados da década de 1970, a trajetória predominante é de redução da diversificação produtiva. Nesse gráfico, a diversificação é entendida como a situação na qual as empresas possuem, pelo menos, duas linhas de produtos, sendo os fluxos de caixa dos diferentes negócios não relacionados.

O fato é que uma parcela considerável das diversificações produtivas promovidas pelas empresas norte-americanas foi mal-sucedida. Nesse contexto, Porter (1998, p. 237), que talvez seja uma das principais contribuições da área de estratégia que relacionam diversificação e desempenho, ressalta:

(...) a história das estratégias corporativas tem sido lamentável. Estudei o passado de diversificação de 33 grandes empresas de prestígio nos Estados Unidos no período de 1950 a 1986 e descobri que a maioria delas tinha revendido, mais do que conservado, as aquisições que haviam feito. As estratégias corporativas da maioria das empresas dissiparam, ao invés de criarem, valor para os acionistas.

\section{Figura 1: Evolução da Diversificação Produtiva nos Estados Unidos} (1960-2000)

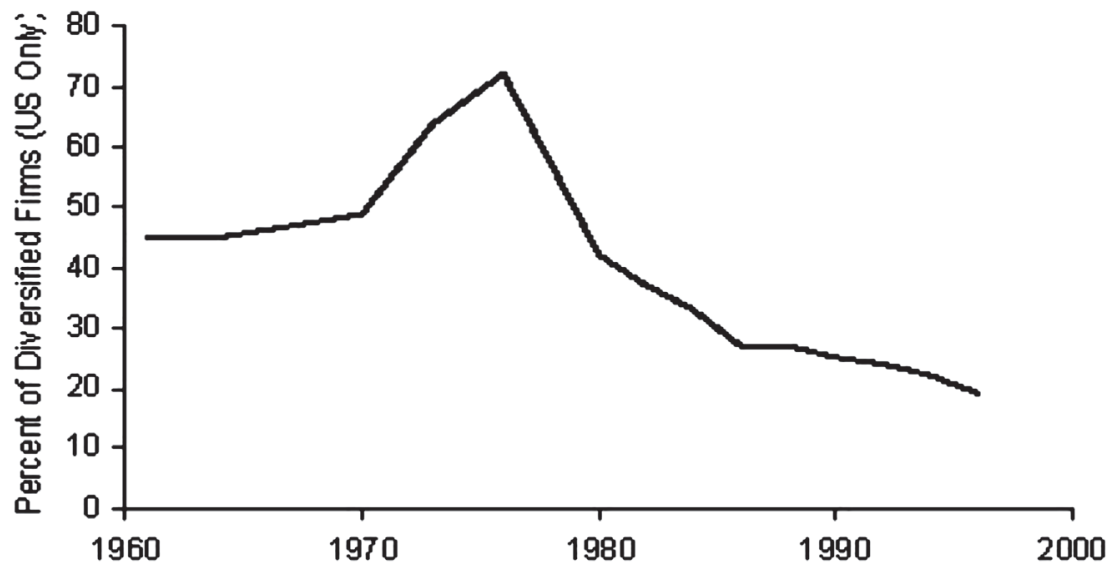

Fonte: Servaes (2002 como citado em Moreira \& Planellas, 2003, p. 2). 
Vários aspectos acerca do desempenho insatisfatório por parte das empresas norte-americanas que optaram pela diversificação - em particular de cunho conglomerado - vêm sendo destacados. Um deles refere-se ao conflito de agência, que é discutido no trabalho seminal de Jensen e Meckling (1976); outros estudos, como os de Lang e Stulz (1994), Berger e Ofek (1996) e Servaes (1996), indicam que as firmas diversificadas possuem um desconto substancial no seu valor, em comparação ao valor imputado àquelas empresas mais focalizadas. Esses trabalhos enquadram-se predominantemente na área de finanças. Assim, pode-se alegar que a discussão entre diversificação e desempenho tem recebido contribuições tanto da literatura de estratégia empresarial, quanto de finanças, o que, aliás, pode alimentar a controvérsia acerca do tema. Esta sucinta revisão da literatura tenta resgatar os resultados principais dessas duas linhas de pesquisa, por acreditar que um enfoque multidisciplinar é o mais adequado para lidar com o tema.

Levando em conta uma estrutura de capital mais pulverizada, alega-se que, se um dos objetivos da diversificação seria a menor vulnerabilidade em face de um mercado específico, isto deveria ser realizado pelos acionistas e não pelas empresas. Reforçando o argumento, quase sempre os acionistas conseguiriam diversificar a um custo menor, comparativamente a um grupo empresarial, já que poderiam comprar ações a preço de mercado, evitando os elevados sobrepreços (prêmios de controle) das aquisições de companhias (Porter, 1998, p. 246).

Nesse contexto, destaca-se o mercado de controle corporativo. Caso a companhia apresente resultados insatisfatórios, os preços das ações tendem a reduzir-se. Considerando que a propriedade dessas ações seja dispersa, a firma corre o risco de sofrer um take-over. Ou seja, uma oferta pública de compra de ações à revelia da direção da empresa. $\mathrm{O}$ risco de take-over seria maior no caso de empresas com alta diversificação produtiva, uma vez que, no mercado de capitais, essas companhias tenderiam a ser precificadas com 'desconto' da ordem de 5 a 15\% (Moreira \& Planellas, 2003, p. 3). A maior dificuldade em estimar os fluxos de caixa, quando uma companhia é altamente diversificada, é um dos motivadores do chamado 'desconto da diversificação conglomerada'. Se o todo vale menos que a soma das partes, muitas vezes a intenção dos promotores do take-over é revender partes do conglomerado, reduzindo os custos efetivos da aquisição. Essas considerações reforçam a visão de que a diversificação produtiva acarreta impactos negativos sobre o valor das empresas e, por conta disto, as empresas deveriam concentrar suas atividades produtivas em torno de suas competências essenciais, abordagem debatida mais recentemente por Best, Hodges e Lin (2004, pp. 235-249) e por Singh, Mathur e Gleason (2004, pp. 489-526).

Na ótica de Singh et al. (2004), a diversificação não é predominantemente uma estratégia de redução de performance, para esses autores não existe desconto no 
valor da firma a partir da diversificação; e mais ainda: as firmas mudam de estratégia de diversificação, tanto mais como para menos focalizada, baseando-se essencialmente nas percepções dos gerentes, quanto a estratégias que possam gerar ganhos para os acionistas. Ademais, Prahalad e Hamel (1990) argumentam que uma abordagem "de dentro para fora" da empresa seria caracterizada pela definição das estratégias competitivas a partir de uma melhor compreensão das possibilidades estratégicas passíveis de operacionalização, bem como sustentação a partir dos recursos da firma. Adiversificação é menos uma resposta às imperfeições estruturais dos mercados e mais um mecanismo organizacional para capturar rendimentos que são tornados viáveis, tendo em vista os ativos da empresa.

Mas Moreira e Planellas (2003, pp. 3-4) destacam a existência de três modelos principais que buscam relacionar diversificação produtiva e desempenho empresarial: 'linear', 'intermediário', e 'relação curvilínea' ou 'modelo U invertido' (Figura 2). De acordo com o primeiro, a diversificação e o desempenho são positivamente relacionados. Isto seria decorrente das vantagens do poder de mercado e da eficiência do mercado (de capitais) interno. Benefícios adicionais são atribuídos à diminuição do risco (teoria do portfólio) e da exploração de ativos específicos. Por exemplo, as vantagens do poder de mercado derivam da possibilidade de utilizar subsídios cruzados entre mercados distintos. $\mathrm{O}$ argumento da eficiência do mercado interno é baseado no fato de que empresas diversificadas possuem acesso tanto ao capital interno quanto externo, o que permite uma utilização mais eficiente do funding (Pawaskar, 1999 como citado em Moreira \& Planellas, 2003, p. 3).

\section{Figura 2: Modelos Relacionando Grau de Diversificação e Desempenho}
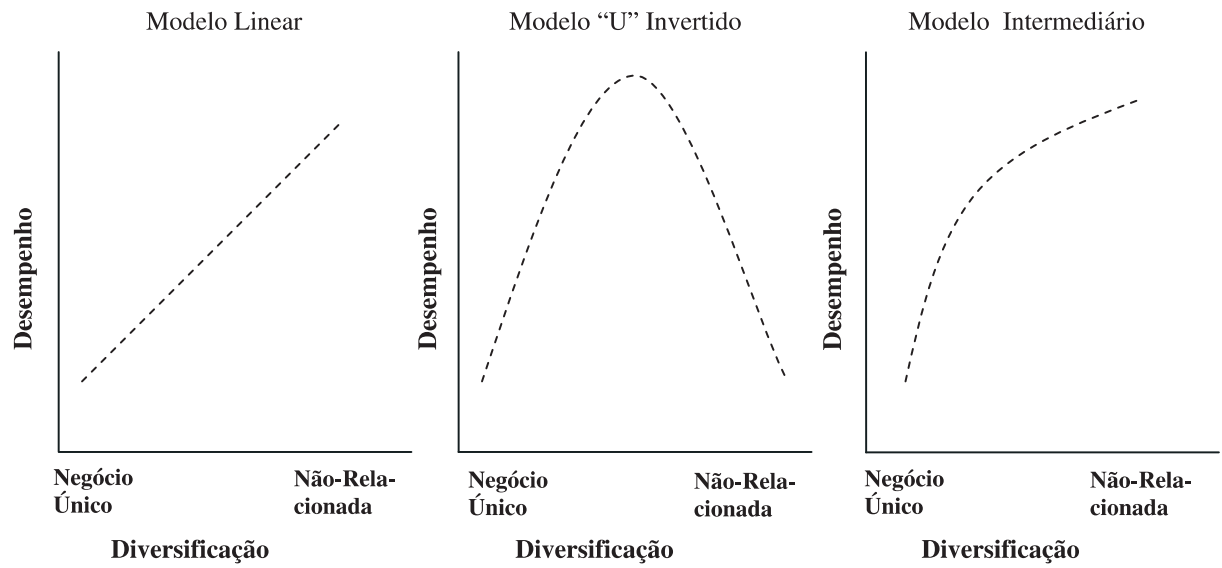

Fonte: Moreira e Planellas (2003, p. 6). 
O modelo intermediário baseia-se no pressuposto de que as empresas são incapazes de explorar as sinergias do portfólio a partir de um determinado grau de diversificação. Ele postula que não existe benefício adicional da diversificação relacionada (ou concêntrica) comparativamente à diversificação não-relacionada (conglomerada). Markides e Williamson (1997) enfatizam e contestam a literatura que afirmam a superioridade da diversificação concêntrica em face da diversificação conglomerada. Embora reconheçam que as economias de escopo constituem importante benefício da diversificação concêntrica, destacam que o aspecto crucial é criar e acumular novos ativos estratégicos de modo mais rápido e barato do que os competidores.

O modelo 'U invertido' é mais influente na literatura norte-americana. Este modelo sugere que o desempenho melhoraria até determinado ponto do grau de diversificação, a partir do qual ele começaria a decrescer. Nesse modelo, a fïma depara-se com um limite em relação à diversificação que pode ser eficientemente gerenciável, sendo que existiriam benefícios marginais de diversificar até certo ponto, a partir do qual os custos marginais passariam a ser predominantes. Mais ainda, deduz-se que a diversificação relacionada seria melhor em termos de desempenho, seja em comparação a uma alta especialização (focalização), seja em face de uma diversificação conglomerada ou não-relacionada (Hitt et al., 2001, p. 256).

\section{Estratégias de Diversificação em Países Emergentes}

De acordo com Lins e Servaes (2002), os trabalhos que estudam a relação entre diversificação produtiva e desempenho em países emergentes são ainda pouco numerosos e os resultados não conclusivos. Por conta disto, nesta subseção examina-se, com algum grau de detalhamento, os poucos artigos que se dedicaram a esta temática.

Em estudo recente, Nachum (2004, pp. 275-294) discorre acerca das diversificações geográfica e produtiva em países em desenvolvimento. A partir de um conjunto de 345 empresas, das quais 61 latino-americanas (incluindo 16 brasileiras), o estudo sugere a existência de uma relação significativa entre diversificação e desempenho, apesar de esta relação variar entre as diferentes regiões estudadas.

Em relação aos países em desenvolvimento da América Latina, Khanna e Palepu (1998) analisaram o desempenho dos grandes grupos chilenos. Nesse estudo, foram consideradas 34 empresas afiliadas a dez grupos econômicos, além de 80 firmas independentes, ao longo do período 1988 a 1996. Três foram os principais resultados obtidos pelos autores. Primeiro, a rentabilidade do ativo 
total, no Chile, foi em média, maior para empresas que eram parte de grupos econômicos do que para firmas independentes. Isto não poderia ser associado ao exercício de rent-seeking, em função do baixo nível de corrupção observado no Chile. Segundo, não se encontrou evidência suficiente para ratificar a hipótese de que os benefícios de afiliação a um grupo econômico, baseado em diversificação conglomerada, tenderiam a diminuir com o passar do tempo. Terceiro, identificou-se uma relação curvilínea (no formato de ' $U$ '), relacionando o grau de diversificação do grupo e o valor das empresas. Assim, a rentabilidade da firma regrediria, se ela participasse de grupo econômico com pouca diversificação até certo limiar, quando então passaria a ter evolução positiva. Esta curva em ' $U$ ' seria consistente com a existência de custos fixos de intermediação, ou seja, somente grupos que excedessem determinado nível de diversificação conglomerada teriam incentivos para investir em sistemas que capitalizassem os benefícios potenciais dos mercados internos de trabalho e capital. Mais importante, o formato da curva seria o oposto do verificado em economias desenvolvidas, como os Estados Unidos.

Ainda analisando o caso chileno, Espinoza e Maquieira (2005), em estudo empreendido com 52 empresas no período de 1995 a 2002, não encontram uma relação conclusiva sobre a base das hipóteses propostas na literatura. Usando os índices de Herfindahl e Entropia e o número de negócios como variáveis de diversificação e o Q de Tobin como variável de desempenho, os autores, em uma análise de correlação preliminar, concluem que a diversificação é elemento que afeta negativamente o desempenho da empresa. Na análise econométrica empreendida pelos autores, os coeficientes das variáveis associadas à diversificação foram positivos, mas não significativos, o que foi contra a evidência preliminar das correlações. Na metodologia utilizada, Espinoza e Maquieira (2005) ainda dividem as variáveis de diversificação em três faixas distintas, colocando uma variável dummy para cada faixa nos modelos econométricos estimados; e concluem que existem diferenças de desempenho entre as faixas de diversificação; entretanto, para alguns indicadores de diversificação, a relação mostrou-se em forma de 'U' e em outros o modelo 'U invertido' foi mais explicativo.

Moreira e Planellas (2003), por sua vez, investigaram a experiência de empresas industriais mexicanas, no período 1997-2001. A amostra foi composta de 60 companhias, cujo controle acionário era detido por investidores domésticos e a variável de desempenho adotada foi a taxa de crescimento das vendas. A diversificação foi avaliada em termos de linhas de produtos de cada empresa. Eles também encontraram uma relação curvilínea, porém com formato próximo ao de 'U invertido', aproximando-se da evidência predominante na literatura acerca das empresas norte-americanas. Esses 
autores apontam ainda que o preenchimento de lacunas institucionais, aspecto central da argumentação de Khanna e Palepu (1998), poderiam, na verdade, estar mais relacionado ao tamanho da empresa do que ao grau da diversificação.

Khanna e Rivkin (2001) ampliam o escopo de análise para catorze países em desenvolvimento, dos quais cinco latino-americanos (Argentina, Brasil, Chile, México e Peru). A variável de desempenho utilizada foi a rentabilidade, e apenas empresas industriais foram incluídas na amostra. Três questões importantes merecem ser destacadas. Primeiro, não se encontrou evidência acerca de algum desconto de diversificação em onze dos catorze países; pelo contrário, foi mais frequiente a existência de um prêmio de diversificação. Segundo, em três países (Índia, Indonésia e Taiwan), foram constatadas evidências robustas de que empresas filiadas a um grupo econômico apresentaram maior rentabilidade do que companhias independentes. Evidências fracas da superioridade das empresas não-independentes foram encontradas em Israel, África do Sul e Peru. Firmas independentes tiveram melhor desempenho na Argentina, de forma significativa, e no Chile e Filipinas, de modo pouco representativo. No Brasil, Coréia do Sul, México, Tailândia e Turquia, os custos e benefícios da afiliação a um grupo econômico foram equilibrados. Terceiro, em doze dos catorze países, os retornos financeiros de empresas filiadas a um grupo foram mais similares com os das suas 'irmãs' do que com os retornos obtidos por companhias que não eram parte do mesmo grupo. As exceções foram México, e Turquia. Na mesma direção, em sete dos catorze países, a filiação (ou não) a um grupo econômico foi mais importante do que a indústria na explicação da rentabilidade da empresa.

Mendes-da-Silva (2004) analisou a experiência brasileira no que tange à relação entre o grau de diversificação e o desempenho financeiro, para o período 1997-2001. Foram consideradas as empresas industriais, com ações negociadas na Bolsa de Valores de São Paulo. O desempenho foi estimado por meio de três medidas: a taxa de crescimento das vendas líquidas, a rentabilidade do ativo total, e o chamado ‘índice Q de Tobin' (valor de mercado da empresa/valor de reposição de ativos). A diversificação foi mensurada pelo grau de concentração dos três principais produtos da empresa. $\mathrm{O}$ autor conclui que não se constatou uma relação curvilínea entre o grau de diversificação da companhia e seu respectivo desempenho. E em apenas dois anos, e mesmo assim por meio de dois indicadores diferentes, foi observado que o desempenho das empresas diversificadas no Brasil teria sido inferior ao das focalizadas. 


\section{Metodologia da Pesquisa}

\section{Os Dados e Definição Operacional das Variáveis}

Em maio de 2001, havia 459 empresas listadas na Bolsa de Valores de São Paulo; dessas 289 possuíam dados disponíveis no Banco de dados da Economática®. Para o desenvolvimento desta pesquisa, foram consideradas todas as empresas industriais (manufatureiras) com dados disponíveis para, pelo menos, três dos cinco anos estudados (1997 a 2001), resultando em um total de 176 empresas industriais de capital aberto.

Após a coleta e tabulação dos dados, procedeu-se à verificação da existência de valores extremos (outliers), que poderiam prejudicar os resultados da pesquisa. Depois de identificados, os outliers foram retirados do banco de dados, assim como recomendam Hair, Anderson, Tatham e Black (1998). Foi estabelecido um limite para a quantidade de observações retiradas: máximo de $10 \%$ das observações válidas. Assim, a distribuição normal dos dados foi assegurada, segundo o procedimento descrito em Hair et al. (1998), que defendem que o escore z para cada distribuição de freqüência, calculado segundo a equação [1], deve permanecer no intervalo de $-2,58$ a $+2,58$.

$$
Z_{s}=\frac{S}{\sqrt{\frac{6}{N}}}
$$

Onde, S é o valor da estatística de assimetria da distribuição de probabilidade de uma variável, e $\mathrm{N}$ representa o tamanho da amostra.

Os dados referentes às variáveis de desempenho foram coletados no Banco de dados da Economática ${ }^{\circledR}$ (2004) e os relativos às variáveis de diversificação foram coletados nos Relatórios de Informações Anuais [IAN], que as empresas remetem anualmente à Comissão de Valores Mobiliários [CVM].

\section{Variáveis de Desempenho}

As métricas usadas para aferir o desempenho ainda não são unânimes no meio acadêmico. Dentre as mais empregadas em estratégia empresarial, de acordo com Barney (1997), quatro categorias se destacam: a) a sobrevivência; b) os indicadores contábeis de desempenho; c) as medidas de criação de valor para os stakeholders e; d) as medidas de valor presente líquido. Nesta pesquisa optouse por três indicadores contábeis de desempenho, que se encontram definidos na 
Tabela 1: i) crescimento da receita líquida por ano (CV), conforme utilizado em estudos sobre diversificação por Bhagat e Black (1999); Parada e Planellas (2003) e Moreira e Planellas (2003); ii) rentabilidade do ativo total (ROA) e; iii) rentabilidade do patrimônio líquido (RPL). Esses dois últimos indicadores são sugeridos por Anderson, Bates, Bizjak e Lemmon (2000) e também por Mendesda-Silva e Magalhães Filho (2004).

Tabela 1: Descrição das Variáveis de Desempenho

\begin{tabular}{|c|c|}
\hline Variável & Descrição \\
\hline $\mathrm{CV}$ & $\begin{array}{l}\text { Taxa de crescimento da receita líquida ao ano calculada por: } \\
\qquad C V=\frac{\text { Vendas }_{i t+1}-\text { Vendas }_{i t}}{\text { Vendas }_{i t}}\end{array}$ \\
\hline ROA & $\begin{array}{l}\text { Rentabilidade do ativo total no ano t expressa por: } \\
\qquad R O A_{i t}=\frac{L L_{i t}}{A_{i t}} \\
\text { Onde, } L L_{i t} \text { é o lucro líquido da empresa i no ano t e } A_{i t} \text { é o } \\
\text { valor contábil do seu ativo total no mesmo período. }\end{array}$ \\
\hline RPL & $\begin{array}{l}\text { Rentabilidade do patrimônio no ano t formulada como: } \\
\qquad R P L_{i t}=\frac{L L_{i t}}{P L_{i t}} \\
\text { Onde, } P L_{i t} \text { expressa o valor contábil do patrimônio líquido da } \\
\text { empresa i no ano t. }\end{array}$ \\
\hline
\end{tabular}

Conforme Moreira e Planellas (2003) e Parada e Planellas (2003), a análise da relação entre a diversificação produtiva e o desempenho alcança maior consistência, quando a amostra analisada é dinâmica, em se tratando de desempenho, pois nesses termos pode-se observar dentre aquelas empresas que tiveram desempenho, quais eram mais ou menos diversificadas. Nesse sentido, depois de excluídos os outliers, procedeu-se também à exclusão das observações de nenhum desempenho, ou seja, permaneceram na amostra as observações cujos valores para as variáveis de desempenho eram positivos $(\mathrm{CV}>0, \mathrm{ROA}>0$ e RPL >0). A Tabela 2 apresenta, depois dos ajustes na amostra, as observações e o número de empresas restantes para análise de cada modelo. 


\section{Variáveis de Diversificação}

Anderson et al. (2000) e Khanna e Palepu (1998) sugerem o Índice HerfindahlHirschman (HHI) de concentração das vendas realizadas com os principais produtos da empresa, para estimar a diversificação produtiva. Na pesquisa tal índice foi calculado usando-se os três principais produtos, segundo a equação [2], assim como também utilizaram Mendes-da-Silva e Magalhães Filho (2004).

$\mathrm{HHI}=\sum_{\mathrm{i}=1}^{3}\left(\frac{\mathrm{p}_{\mathrm{i}}}{\mathrm{p}} \times 100\right)^{2}$

Onde, pi é o valor das vendas líquidas realizadas com um produto e p é o valor total das vendas líquidas da empresa i, no ano t. O valor de HHI é maximizado, quando a participação de um único produto no faturamento da empresa atinge $100 \%$ e nesses termos HHI $=10.000$. Quando os produtos têm participação igualitária, o índice assume o menor valor, $\mathrm{HHI}=1 / \mathrm{n}$.

\section{Tabela 2: Característica da Amostra Depois dos Ajustes}

\begin{tabular}{|c|c|c|c|c|}
\hline Modelo & $\begin{array}{c}\text { Variável } \\
\text { Independente }\end{array}$ & Variável Dependente & $\mathbf{N}^{0}$ Empresas & $\mathbf{N}^{\circ}$ Observações \\
\hline MOD1 & \multirow{3}{*}{ HHI } & $\mathrm{CV}$ & 129 & 344 \\
\hline MOD2 & & ROA & 114 & 303 \\
\hline MOD3 & & RPL & 112 & 302 \\
\hline MOD4 & \multirow{3}{*}{$\mathrm{CE}$} & $\mathrm{CV}$ & 135 & 399 \\
\hline MOD5 & & ROA & 120 & 352 \\
\hline MOD6 & & RPL & 116 & 349 \\
\hline
\end{tabular}

Khanna e Palepu (1998) sugerem ainda o cálculo do Coeficiente de Entropia (CE) como medida de concentração das vendas (diversificação). Foram consideradas no presente trabalho também as concentrações de vendas realizadas com os três principais produtos da empresa, que foram calculadas segundo a equação [3].

$$
\mathrm{CE}=\sum_{\mathrm{i}=1}^{3} \mathrm{P}_{\mathrm{i}} \times \log \frac{1}{\mathrm{P}_{\mathrm{i}}}
$$

Onde, pi é participação do produto no faturamento da empresa. O CE corresponde a uma medida que permite comparar as diferenças no tempo, no grau de concentração dos produtos. Quando existir apenas um produto, $\mathrm{CE}=0$; quando todos os produtos apresentam igual participação no faturamento da empresa, a entropia é maximizada e $\mathrm{CE}=\log \mathrm{n}$. No estudo o valor do CE é maximizado, quando $\mathrm{CE}=\log 3 \cong 0,47712$. 
Cabe ressaltar algumas considerações sobre as variáveis CE e HHI. Evidenciouse na tabulação dos dados que, calculando essas variáveis com apenas três produtos, seria suficiente para formar uma boa proxy de diversificação, uma vez que a participação média nas vendas do principal produto na amostra se situou em torno de 65\%; para os dois mais importantes, $83 \%$; e para os três mais importantes, $91 \%$.

\section{Especificação dos Modelos}

Na pesquisa empreendida dispõe-se de observações sobre várias empresas para alguns períodos de tempo, ou seja, a amostra apresenta combinação de séries temporais e dados em corte transversal. A análise empregada comumente também é denominada de análise de painel. A análise de dados em painel apresenta algumas complicações, pois existe o problema de como especificar um modelo estatístico que capte diferenças individuais de comportamento, de modo que possa combinar (ou misturar) todos os dados (informações) para fins de estimação e inferência (Hill, Griffiths, \& Judge, 2003, p. 406). Dessa forma, dois métodos específicos para a análise de dados em painel se destacam: o método estimado por Mínimos Quadrados Ordinários [MQO] com Efeitos Fixos [EF] e o método estimado por MQO com Efeitos Aleatórios [EA].

$\mathrm{O}$ método de EF pode ser modelado de três formas: 1) regressões com EF entre entidades; 2) regressões com EF temporais; e 3) regressões com EF entre entidades e temporais (Stock \& Watson, 2004, pp. 185-201). Os modelos com EF entre entidades permitem controlar variáveis não observadas que diferem de uma entidade para outra, tais como o tamanho e o setor de atuação das empresas, mas que não variam ao longo do tempo. Os modelos de EF temporais permitem controlar variáveis que se alteram ao longo do tempo, como a fase de crescimento econômico setorial, mas que não variam entre as entidades. Porém, se algumas variáveis omitidas são constantes ao longo do tempo, mas variam entre as entidades, enquanto outras são constantes entre entidades, mas variam ao longo do tempo; então se torna apropriado estimar os modelos com EF entre entidades e temporais. A grande vantagem do método de EF é que são controlados alguns tipos de variáveis omitidas sem observá-las de fato.

O trabalho optou por fazer uso do EF entre entidades e temporal, uma vez que se assume a existência de uma complexidade de variáveis que influenciam o desempenho de uma empresa, assim como vários fatores podem ter contribuído para sua alteração ao longo dos anos. Desta forma, a relação estimada entre o desempenho e a diversificação torna-se imune ao viés da variável omitida que é constante ao longo do tempo ou entre as entidades. A modelagem dos EF entre entidades e temporal é obtida por meio da introdução de variáveis binárias (dummy) 
que permitam ao termo de intercepto variar. Generaliza-se o modelo de EF entre entidades e ao longo do tempo para as varáveis do trabalho da seguinte forma:

$Y_{i t}=\alpha+\beta X_{i t}+\gamma_{2} W_{2 i}+\gamma_{3} W_{3 i}+\ldots+\gamma_{N} W_{N i}+\delta_{2} Z_{i 2}+\delta_{3} Z_{i 3}+\ldots+\gamma_{T} W_{T i}+\varepsilon_{i t}$

Onde,

$Y_{i t}=$ medidas de desempenho CV, ROA e RPL da empresa i no ano t.

$X_{i t}=$ medidas de concentração $\mathrm{HHI}$ e CE da empresa i no ano t, sendo que tais medidas podem tomar a forma quadrática, cúbica e logarítmica, dependendo da forma funcional do modelo.

$$
\begin{aligned}
& W_{i t}=\left\{\begin{array}{l}
1 \text { para a } i \text { - ésima entidade, } i=1,2, \ldots, \mathrm{N} \\
0 \text { caso contrário }
\end{array}\right. \\
& Z_{i t}=\left\{\begin{array}{l}
1 \text { para a } t \text { - ésimo período, } t=1997, \ldots, 2001 . \\
0 \text { caso contrário }
\end{array}\right.
\end{aligned}
$$

Destaque-se que $\mathrm{N}$ nas variáveis dummy das entidades pode assumir diferentes valores, dependendo do modelo: $\mathrm{N}=129$ se MOD1; $\mathrm{N}=114$ se MOD2; $\mathrm{N}=112$ se $\mathrm{MOD} 3 ; \mathrm{N}=135$ se $\mathrm{MOD} 4, \mathrm{~N}=120$ se $\mathrm{MOD}$; e $\mathrm{N}=116$ se MOD6.

Ademais, a inclusão dos EF apresenta dois problemas: resulta numa perda de graus expressivos de liberdade, se $\mathrm{N}$, o número de entidades, for grande; e sugere a falta de conhecimento do modelo (Maddala, 2003, p. 309). Nesse sentido, torna-se natural descrever a falta de conhecimento com a utilização do termo de perturbação $\left(\varepsilon_{i t}\right)$, surgindo assim os modelos com Efeitos Aleatórios [EA]. Esses modelos combinam dados em corte transversal e de séries temporais, supondo-se que os termos de erro podem estar correlacionados ao longo do tempo e ao longo das entidades (Pindyck \& Rubinfeld, 2004, p. 292). O método de EA para as variáveis da pesquisa apresenta-se da seguinte forma:

$Y_{i t}=\alpha+\beta X_{i t}+\varepsilon_{i t}$

$\varepsilon_{i t}=u_{i t}+v_{i t}+w_{i t}$

Onde,

$Y_{i t}$ e $X_{i t}$ definidos conforme no modelo de EF (equação [4]) 


$$
\begin{aligned}
& u_{i t} \sim N\left(0, \sigma_{u}^{2}\right)=\text { componente de erro pelos dados em corte transversal } \\
& v_{i t} \sim N\left(0, \sigma_{v}^{2}\right)=\text { componente de erro pelos dados em séries temporais } \\
& w_{i t} \sim N\left(0, \sigma_{w}^{2}\right)=\text { componente de erro combinado }
\end{aligned}
$$

O modelo de EA pode ser estimado como uma regressão de Mínimos Quadrados Generalizados [MQG]. A estimação pondera as observações inversamente a suas variâncias.

Cabe destacar que se poderia usar o teste de especificação de Hausman para escolher entre os métodos EF e EA, o mais eficiente. Entretanto os resultados dos testes de Hausman devem ser interpretados com cautela, pois são sensíveis aos desvios em relação às suposições de homocedasticidade e não autocorrelação dos termos de erro. Por essa razão, os coeficientes estimados por EF e EA serão apresentados para todos os modelos.

\section{Resultados}

Buscou-se estimar para as regressões, conforme os três principais modelos teóricos que sustentam a associação entre diversificação e desempenho (modelo linear, modelo curvilíneo e modelo intermediário), as seguintes formas funcionais: linear, quadrática, cúbica e logarítmica. Nesse sentido, considerando os seis modelos (MOD1, ..., MOD6) e os dois métodos priorizados, EF e EA, estimouse um total de 48 regressões. As próximas quatro tabelas apresentam os resultados de cada regressão, excluindo para os modelos com EF a análise dos coeficientes das variáveis dummy, por motivo de irrelevância e economia de espaço.

É comum evidenciar os sinais dos coeficientes esperados conforme a teoria, porém como apresentado na revisão bibliográfica, as evidências empíricas mostram-se inconclusivas sobre a relação entre diversificação produtiva e desempenho, de tal forma que, indo ao encontro do objetivo desta pesquisa, procurar-se-á a forma funcional mais significativa e com melhor grau de ajuste, conforme metodologia empregada.

Todas as regressões foram estimadas com erros-padrão consistentes quanto à heterocedasticidade, segundo White. Em relação às estimativas por EF, o Teste Wald para as variáveis dummy das entidades apresentou significância estatística ao nível de $1 \%$ em todas regressões, rejeitando que no conjunto os coeficientes das dummy são iguais a zero. Da mesma forma, em relação às dummy temporais, 
rejeitou-se em todas as regressões cujos coeficientes são iguais a zero, ou seja, os modelos estimados com EF se justificam isoladamente. Os p-values dos testes Wald apresentados nas Tabelas 3 e 4 são apenas para as variáveis $\mathrm{X}_{i t}$, não incluindo as variáveis dummy entre entidades e temporais. Ainda em relação aos modelos com EF, realizou-se o teste de autocorrelação (AR Test) de primeira ordem e segunda ordem, rejeitando em todas as regressões a hipótese de autocorrelação da ordem.

Tabela 3: Resultados dos Modelos com a Variável Independente HHI e Efeitos Fixos

\begin{tabular}{|c|c|c|c|c|c|c|c|c|}
\hline$Y_{i t}$ & Modelo & $\begin{array}{c}\text { Forma } \\
\text { Funcional }\end{array}$ & Constante & HHI & $\mathrm{HHI}^{2}$ & $\mathrm{HHI}^{3}$ & $\mathrm{Ln}(\mathrm{HHI})$ & $\begin{array}{c}\operatorname{Prob}\left(\mathbf{X}^{2}\right) \\
\text { Wald Test }\end{array}$ \\
\hline \multirow{4}{*}{$\mathrm{CV}$} & \multirow{4}{*}{ MOD1 } & Linear & $61,8044 *$ & $-0,0012$ & & & & 0,504 \\
\hline & & Quadrática & $59,2688^{*}$ & $-0,0002$ & $-9,5 e-008$ & & & 0,780 \\
\hline & & Cúbica & $58,227^{*}$ & $0,0093^{* *}$ & $-2,3 e-006^{*}$ & $1,4 \mathrm{e}-01^{*}$ & & 0,001 \\
\hline & & Logarítmica & 85,38 & & & & $-1,8538$ & 0,780 \\
\hline \multirow{4}{*}{ ROA } & \multirow{4}{*}{ MOD2 } & Linear & $-7,0238^{*}$ & $-2,1 e-005$ & & & & 0,902 \\
\hline & & Quadrática & $-4,4898 * *$ & $-0,001 *$ & $1,01 \mathrm{e}-007 *$ & & & 0,001 \\
\hline & & Cúbica & $-5,8062$ & $0,005^{*}$ & $-1,41 \mathrm{e}-006^{*}$ & $1 \mathrm{e}-01 *$ & & 0,000 \\
\hline & & Logarítmica & 0,0000 & & & & $-0,1415$ & 0,806 \\
\hline \multirow{4}{*}{ RPL } & \multirow{4}{*}{ MOD3 } & Linear & 70,4327 & $-0,0059$ & & & & 0,237 \\
\hline & & Quadrática & 70,4773 & 0,0056 & $-1,19 \mathrm{e}-006$ & & & 0,009 \\
\hline & & Cúbica & $-200,141^{*}$ & $0,047 * * *$ & $-1,1 \mathrm{e}-005^{* *}$ & $6,5 \mathrm{e}-01 * *$ & & 0,000 \\
\hline & & Logarítmica & 0,0000 & & & & $-14,3238$ & 0.196 \\
\hline
\end{tabular}

Significância: *1\%;**5\%;***10\%.

Tabela 4: Resultados dos Modelos com a Variável Independente CE e Efeitos Fixos

\begin{tabular}{|c|c|c|c|c|c|c|c|c|}
\hline$Y_{i t}$ & Modelo & $\begin{array}{c}\text { Forma } \\
\text { Funcional }\end{array}$ & Constante & $\mathbf{C E}$ & $\mathrm{CE}^{2}$ & $\mathrm{CE}^{3}$ & $\operatorname{Ln}(C E)$ & $\begin{array}{r}\text { Prob }\left(\mathbf{X}^{2}\right) \\
\text { Wald Test }\end{array}$ \\
\hline \multirow{4}{*}{$\mathrm{CV}$} & \multirow{4}{*}{ MOD4 } & Linear & 24,2059 & 16,6030 & & & & 0,545 \\
\hline & & Quadrática & $30,8170 * *$ & $-39,2094$ & 98,2622 & & & 0,251 \\
\hline & & Cúbica & $49,6274 *$ & $-537,65^{*}$ & $2347,35^{*}$ & $-2826,48^{*}$ & & 0,000 \\
\hline & & Logarítmica & $35,8358 *$ & & & & 4,4016 & 0,384 \\
\hline \multirow{4}{*}{ ROA } & \multirow{4}{*}{ MOD5 } & Linear & $4,9 *$ & 0,5636 & & & & 0,761 \\
\hline & & Quadrática & $3,8^{*}$ & $-44,548 *$ & $79,1264^{*}$ & & & 0,000 \\
\hline & & Cúbica & $4,9 *$ & $-16,6848$ & $-51,1476$ & 166,844 & & 0,000 \\
\hline & & Logarítmica & $9,188^{*}$ & & & & $0,7491 * *$ & 0,010 \\
\hline \multirow{4}{*}{ RPL } & \multirow{4}{*}{ MOD6 } & Linear & 8,3 & 108,195 & & & & 0,261 \\
\hline & & Quadrática & $10,6^{*}$ & 140,272 & $-56,2554$ & & & 0,117 \\
\hline & & Cúbica & $6,6^{*}$ & $-1167 * * *$ & $6041 * * *$ & $-7810 * * *$ & & 0,002 \\
\hline & & Logarítmica & 124,824 & & & & 27,6435 & 0,162 \\
\hline
\end{tabular}

Significância: $* 1 \% ; * * 5 \% ; * * * 10 \%$. 
Para estimar os modelos com EA, fez-se uso do método dos MQG, utilizando os resíduos para estimar as variâncias. Para todas as regressões foram também testadas as hipóteses de autocorrelação pelo teste de Breusch-Godfrey, que rejeita tal hipótese para todos os modelos. Mesmo estimando os modelos com errospadrão consistentes quanto à heterocedasticidade, testou-se a hipótese de heterocedasticidade pelo método de White, o qual rejeitou a mesma hipótese para todos os modelos. Fazendo a análise das formas funcionais estimadas, conforme regressões apresentadas nas Tabelas de 3 a 6 , encontram-se os seguintes resultados:

Tabela 5: Resultados dos Modelos com a Variável Independente HHI e Efeitos Aleatórios

\begin{tabular}{|c|c|c|c|c|c|c|c|c|}
\hline$Y_{i t}$ & Modelo & $\begin{array}{c}\text { Forma } \\
\text { Funcional }\end{array}$ & Constante & HHI & $\mathrm{HHI}^{2}$ & $\mathrm{HHI}^{3}$ & Ln(HHI) & $\begin{array}{c}\text { Prob } \\
\text { (F-statistic) }\end{array}$ \\
\hline \multirow{4}{*}{$\mathrm{CV}$} & \multirow{4}{*}{ MOD1 } & Linear & $23,4797^{*}$ & 0,0005 & & & & 0,296 \\
\hline & & Quadrática & $21,3012 *$ & 0,0015 & $-8,66 \mathrm{e}-08$ & & & 0,534 \\
\hline & & Cúbica & $47,4641 *$ & $-0,0192 *$ & $4,42 \mathrm{e}-06^{*}$ & $-2,74 \mathrm{e}-10$ & & 0,022 \\
\hline & & Logarítmica & 12,4993 & & & & 1,6592 & 0,499 \\
\hline \multirow{4}{*}{ ROA } & \multirow{4}{*}{ MOD2 } & Linear & $4,6982 *$ & 0,0001 & & & & 0,134 \\
\hline & & Quadrática & $4,0326^{*}$ & 0,0004 & $-2,57 \mathrm{e}-08$ & & & 0,275 \\
\hline & & Cúbica & $6,159^{*}$ & $-0,0011$ & $3,26 \mathrm{e}-07$ & $-2,13 \mathrm{e} 11$ & & 0,299 \\
\hline & & Logarítmica & $-0,2763$ & & & & 0,6952 & 0,176 \\
\hline \multirow{4}{*}{ RPL } & \multirow{4}{*}{ MOD3 } & Linear & $14,6135^{*}$ & 0,0003 & & & & 0,754 \\
\hline & & Quadrática & 3,0123 & 0,0054 & $-4,47 e-07$ & & & 0,575 \\
\hline & & Cúbica & $32,82 * * *$ & $-0,0176$ & $4,5 \mathrm{e}-06^{* * *}$ & $-3 e-10 * * *$ & & 0,361 \\
\hline & & Logarítmica & 5,7726 & & & & 1,2689 & 0,807 \\
\hline
\end{tabular}

Significância: $* 1 \% ; * * 5 \% ; * * * 10 \%$.

Tabela 6: Resultados dos Modelos com a Variável Independente CE e Efeitos Aleatórios

\begin{tabular}{|c|c|c|c|c|c|c|c|c|}
\hline$Y_{i t}$ & Modelo & $\begin{array}{c}\text { Forma } \\
\text { Funcional } \\
\end{array}$ & Constante & CE & $\mathrm{CE}^{2}$ & $\mathrm{CE}^{3}$ & $\operatorname{Ln}(\mathbf{C E})$ & $\begin{array}{c}\text { Prob } \\
\text { (F-statistic) }\end{array}$ \\
\hline \multirow{4}{*}{$\mathrm{CV}$} & \multirow{4}{*}{ MOD4 } & Linear & $28,8974 *$ & $-9,5254$ & & & & 0,309 \\
\hline & & Quadrática & $22,713^{*}$ & $73,296^{* *}$ & $-171,41 * *$ & & & 0,035 \\
\hline & & Cúbica & $22,3127 *$ & 89,252 & $-257,621$ & 119,989 & & 0,081 \\
\hline & & Logarítmica & $23,6112 *$ & $-2,0686$ & & & & 0,307 \\
\hline \multirow{4}{*}{ ROA } & \multirow{4}{*}{ MOD5 } & Linear & $6,3939 *$ & $-2,4392$ & & & & 0,264 \\
\hline & & Quadrática & $5,6821 *$ & 7,9779 & $-22,1252$ & & & 0,231 \\
\hline & & Cúbica & $5,9015^{*}$ & $-1,591$ & 29,2483 & $-71,004$ & & 0,371 \\
\hline & & Logarítmica & $5,3707 *$ & & & & $-0,1747$ & 0,692 \\
\hline \multirow{4}{*}{ RPL } & \multirow{4}{*}{ MOD6 } & Linear & $14,6079 *$ & 4,2981 & & & & 0,814 \\
\hline & & Quadrática & $9,3591 *$ & 82,069 & $-165,640$ & & & 0,502 \\
\hline & & Cúbica & $12,2762 *$ & $-45,8588$ & 521,740 & $-950,856$ & & 0,594 \\
\hline & & Logarítmica & $18,2506^{*}$ & & & & 1,7461 & 0,645 \\
\hline
\end{tabular}

Significância: $* 1 \% ; * * 5 \%$; ***10\%. 
Linear: nos modelos com EF, tanto para a variável HHI como CE, a relação entre diversificação e desempenho apresentou-se positiva, porém os modelos no conjunto não se apresentaram significativos, conforme teste Wald apresentado nas Tabelas 3 e 4. Para os modelos com EA, com exceção do MOD6, achou-se uma relação negativa, ou seja, à medida que as empresas diversificam, o seu desempenho medido por CV, ROA e RPL diminuem. Entretanto, como nos modelos com EF, nenhum se apresentou significativo.

Quadrática: usando a variável dependente CV, apenas o MOD4 com EA apresentou-se significativo no seu conjunto, ao nível de 5\%, com uma relação na forma de ' $U$ '. Os modelos com a variável dependente ROA (MOD2 e MOD5) mostraram-se significativos, ao nível de 1\%, pelo método de EF, com uma relação de 'U invertido'. Usando a variável dependente RPL, apenas o MOD3 com EF apresentou-se significativo, ao nível de 1\%, com uma relação na forma de 'U'.

- Cúbica: essa forma foi a que mais obteve freqüência de significância. Para os modelos estimados com EF, exceto o MOD5, a relação mostrada na forma de um 'duplo U', apresentou em um primeiro momento um ' $U$ invertido', para posteriormente a curva ascender, ou seja, o desempenho medido pelas variáveis CV, ROA e RPL crescem até um nível de diversificação, para depois cair e posteriormente ascender novamente. Observa-se que apesar de os coeficientes individuais do MOD5 não apresentarem significância, os coeficientes no conjunto apresentam significância ao nível de 1\%, porém com sinal contrário do termo ao quadrado $\left(\mathrm{HHI}^{2}\right)$. Pelo método dos EA, apenas os MOD1 e MOD4 se apresentaram significativos (ao nível de 1\% e 10\%, respectivamente), entretanto a relação de 'duplo U' mostrou-se contrária. O desempenho, medido por CV, em um primeiro momento, reduz-se até um ponto mínimo de diversificação, para posteriormente crescer e, em um terceiro momento, decrescer, ou seja, primeiramente a regressão toma a forma de ' $U$ ' e, posteriormente, a forma de 'U invertido'.

- Logarítmica: apenas o MOD5 com EF apresentou-se significativo, ao nível de $1 \%$, com uma relação positiva.

Cabe ainda ressaltar que não foram apresentados os coeficientes de determinação $\left(\mathrm{R}^{2}\right)$, pois os seus valores não são diretamente comparáveis. As regressões com EF tendem a apresentar $\mathrm{R}^{2}$ alto, porque os efeitos fixos evidentemente respondem por uma grande variação nos dados (Stock \& Watson, 2004, p. 195). Conforme se apresenta na caracterização dos dados, estes não foram escolhidos aleatoriamente. Esse fato contribuiu para que os resíduos de cada regressão não apresentassem a hipótese de normalidade. O teste de normalidade Jarque-Bera dos resíduos rejeitou a hipótese de normalidade em 
todas as regressões, contribuindo possivelmente para que os estimadores de MQO dos coeficientes sejam não confiáveis. Porém, como se considera que a amostra em cada modelo é suficientemente grande, os testes de hipóteses usuais são ainda válidos assintoticamente, ou seja, em amostras grandes (Gujarati, 2000, pp. 314-315).

\section{CONCLUSÕES}

Existe extensa e crescente literatura mundial sobre estratégias corporativas que abordam essencialmente o relacionamento entre diversificação ou focalização com o desempenho empresarial. Em geral, essa literatura é concentrada em mercados desenvolvidos, particularmente nos Estados Unidos. As conclusões das pesquisas feitas nesses mercados tendem a apoiar uma relação curvilínea ('U invertido') entre a diversificação e desempenho. Segundo essa perspectiva, o desempenho aumenta com a diversificação até certo ponto, após o qual começa a se reduzir, conduzindo à conclusão de que as firmas, em face das restrições, podem administrar eficientemente a diversificação até certo estágio. De forma alinhada com essa perspectiva, as grandes empresas norteamericanas, principalmente a partir da década de 1980, vêm concentrando seus negócios.

Diferentemente para países emergentes, particularmente para América Latina, a literatura é escassa e não unânime sobre a relação entre diversificação e desempenho (Lins \& Servaes, 2002). Nesse sentido, Moreira e Planellas (2003), investigando amostras de empresas industriais mexicanas, encontraram uma relação aproximada de 'U invertido', à semelhança da evidência predominante na literatura norte-americana. Moreira e Planellas (2003, p. 14) citam ainda evidências empíricas que parecem apoiar um efeito positivo constante entre a diversificação e o desempenho empresarial nos países emergentes, ou seja, essas pesquisas comprovariam o modelo linear, assim como também encontram Espinoza e Maquieira (2005). Esses últimos, além desse achado, também encontraram apoio empírico que sustenta o modelo em ' $U$ ' e o modelo de ' $U$ invertido', permanecendo, de modo geral, o estudo dos autores, inconcluso sobre a base das hipóteses propostas na literatura.

De outro lado, Khanna e Palepu (1998), analisando o desempenho dos grandes grupos chilenos, identificaram uma relação curvilínea no formato de 'U', condizente com a existência de custos fixos de intermediação e oposto do verificado em economias desenvolvidas, como os Estados Unidos. À luz dessa perspectiva, somente grupos que excedessem determinado nível de diversificação 
conglomerada teriam incentivos para investir em sistemas que capitalizassem os benefícios potenciais dos mercados internos de trabalho e capital.

O objetivo desta pesquisa foi estudar a relação existente entre a estratégia de diversificação e o desempenho empresarial e, por conseguinte, buscar evidências empíricas sobre o modelo que melhor possa explicar essa relação na indústria brasileira. Usando as variáveis de desempenho: Retorno sobre o Patrimônio Líquido [RPL], Retorno sobre o Ativo [ROA] e Crescimento das Vendas [CV]; e as variáveis de diversificação: Índice Herfindahl-Hirschman [HHI] e Coeficiente de Entropia [CE]; para um painel de 176 empresas industriais brasileiras, listadas na Bolsa de Valores de São Paulo nos anos de 1997 a 2001, procedeu pelo método dos Mínimos Quadrados Ordinários [MQO], metodologia especifica para análise de dados em painel. Por meio dos procedimentos de Efeitos Fixos [EF] entre entidades e temporais e dos Efeitos Aleatórios [EA], conclui-se pela existência da relação curvilínea entre a diversificação e o desempenho. Contudo a relação mostrou-se indefinida, não sendo possível concluir se ocorre sob a forma de ' $U$ ' ou de 'U invertido'.

Para a forma quadrática, que efetivamente traduz a relação curvilínea em forma de 'U', das 12 equações estimadas, quatro mostraram significância com nível máximo de 5\%; entretanto duas apresentaram a forma de ' $U$ ' e duas a forma de 'U invertido'. Para a forma cúbica, que se apresenta como aproximação do modelo curvilíneo, das doze equações estimadas, oito mostraram significância com nível máximo de $10 \%$; todavia novamente cinco formas curvilíneas apresentam o formato de ' $U$ invertido' e três de ' $U$ '. Das equações estimadas com o modelo linear, nenhuma apresentou significância estatística, sendo que apenas uma se mostrou significativa ao nível de $1 \%$ para o modelo intermediário.

Uma das particularidades desta pesquisa em relação a outras existentes nos mercados emergentes, em particular no Brasil, diz respeito aos procedimentos empregados. O procedimento dos $\mathrm{MQO}$ por EF entre entidades e temporais controla alguns tipos de variáveis omitidas sem observá-la de fato. Assumindo que existe uma complexidade de variáveis que influenciam o desempenho de uma empresa, assim como vários fatores podem ter contribuído para que alterasse o desempenho ao longo dos anos, esse procedimento torna-se apropriado, sendo a relação estimada entre a diversificação e o desempenho imune ao viés de variável omitida que é constante, ou ao longo do tempo ou entre as entidades. Da mesma forma, como existe a falta de conhecimento da relação existente entre a diversificação e o desempenho (indefinida como apresenta a revisão da literatura), torna-se apropriado o uso do procedimento de EA.

Todavia quatro limitações precisam ser ressaltadas, sendo duas inerentes aos dados e duas em relação à metodologia. Quanto às primeiras: a) as métricas de 
desempenho são obtidas de dados contábeis e dessa forma possuem deficiências relacionados à fidedignidade; b) as métricas de diversificação estão relacionadas à diversificação produtiva e assume-se que sejam baseados em outros trabalhos empíricos e que elas sejam uma boa proxy das variáveis de diversificação de negócios. No que tange às limitações relacionadas à metodologia: c) como se utilizou uma amostragem por julgamento, os resíduos das equações estimadas não apresentaram distribuição normal, conforme teste de Jarque-Bera, valendo os resultados obtidos assintoticamente; e d) como consequiência do julgamento feito na amostragem e da falta de informações disponíveis para alguns anos do estudo, o painel apresentou-se desequilibrado, como conseqüência de ter perdido alguma informação na análise pretendida.

Em suma, as lições recebidas da revisão da literatura para os gestores na América Latina e as recebidas da presente pesquisa para os gestores no Brasil resumem que não há evidência suficiente para afirmar que a rentabilidade da firma regrida, se ela é pouco diversificada até certo limiar, quando então passa a ter evolução positiva ('U'), assim como também não se tem evidência empírica suficiente para conjeturar que os gestores podem administrar eficientemente a diversificação até certo montante ('U invertido').

Esses apontamentos mostram, de um lado, a inconclusão em relação à associação existente entre a diversificação produtiva e o desempenho na América Latina, e em especial no Brasil. Isto representa certo distanciamento em face da literatura predominante quanto ao mesmo tema nos Estados Unidos. De outro, isto conduz a uma convergência, pois tanto nos países desenvolvidos quantos nos emergentes existiriam pontos em que os custos marginais da diversificação superariam os benefícios marginais (modelo curvilíneo). Portanto este estudo chegou à conclusão de que os modelos linear e intermediário devem ser rejeitados. Reforçando o argumento, os dados não apresentaram aderência suficiente para aceitar a hipótese de que haveria um efeito positivo entre o grau de diversificação e o desempenho empresarial, seja linear seja exponencialmente.

Outro aspecto importante a ser destacado nesta oportunidade é que uma significativa parcela dos estudos teóricos e empíricos acerca da temática da diversificação da firma atribui a decisão por diversificar ou concentrar o negócio da firma às estruturas de governança corporativa, isto é, as relações de agência, bem como os métodos de controle dos problemas dela advindos seriam os principais determinantes da decisão pela diversificação ou concentração do negócio. Porém, na situação das economias desenvolvidas, sobretudo nos Estados Unidos, os conflitos de interesse situam-se entre acionistas e executivos, caracterizando a relação principal-agente. E, no caso brasileiro, tendo em vista especialmente as especificidades das estruturas de propriedades das grandes empresas, os conflitos ocorrem essencialmente entre acionistas majoritários e minoritários. Em outras 
palavras, os aspectos destacados pela literatura como relevantes para impulsionar uma superdiversificação em empresas norte-americanas, pelo menos em princípio, não devem ser os mesmos no Brasil, uma vez que no caso nacional as questões relativas às práticas de governança corporativa inerentes à conciliação de interesses no âmbito da firma possuem particularidades em relação ao caso geral tratado na literatura internacional.

Essas conclusões, aliadas à metodologia aqui empregada, com as ressalvas usuais, parecem vislumbrar um promissor campo de pesquisa. Ademais, como já mencionado, ainda é relativamente incipiente a produção acadêmica acerca desta temática no Brasil e na América Latina. Desse modo, sugere-se que novas pesquisas: a) façam uso de outras medidas de diversificação e desempenho financeiro; b) utilizem medidas de valor, como o 'Q de Tobin'; c) estudem períodos diferentes com amostras compostas por empresas que não tenham suas ações negociadas em Bolsa de Valores; d) incorporem, na metodologia estatística empregada, variáveis de controle, tais como setor, tamanho e tipo de controle patrimonial; e) examinem o desempenho de empresas afiliadas e não-afiliadas aos grupos econômicos.

\section{Artigo recebido em 27.07.2005. Aprovado em 22.11.2005.}

\section{RefERÊNCIAS BibliográficAs}

Anderson, R. C.,

Bates, T. W.,

Bizjak, J. M., \&

Lemmon, M. L. (2000).

Corporate governance and firm diversification.

Financial

Management, 29(1), 5-22.

Barney, J. B. (1997).

Gaining and sustaining competitive advantage. Reading, MA: Addison Wesley Publishing Company.

Berger, P. G., \&

Ofek, E. (1996).

Bustup takeovers of valuedestroying diversified firms. Journal of Finance, 51(4), 1175-1200.
Best, R. W.,

Hodges, C. W., \&

Lin, B. X. (2004).

Does information asymmetry explain the diversification discount? The Journal of Financial Research, 27(2), 235-249.

Bhagat, S., \&

Black, B. (1999).

The uncertain relationship between board composition and firm performance. The Business Lawyer, 54(3), 921-963. 
Comissão de Valores Imobiliários. (2004). Relatórios de informações anuais. Recuperado em 20 setembro, 2004, de http://cvmweb.cvm.gov.br/SWB/ Sistemas/SCW/CPublica/CiaAb/ FormBuscaCiaAb.aspx?TipoConsult=c

Economática ${ }^{\circledR}$. (2004).

Tools for investment análisis (Banco de Dados/2004). São Paulo, SP, Economática Brasil.

Espinoza, C., \&

Maquieira, C. (2005).

Relacion entre valor de la empresa, diversificación y gobierno corporativo. Anais da Assembléia do Conselho Latino-Americano das Escolas de Administração, Santiago do Chile, Chile, 40.

Gujarati, D. N. (2000).

Econometria básica. São Paulo: Makron Books Ltda.

Hair, J. F., Jr.,

Anderson, R. E.,

Tatham, R. L., \&

Black, W. C. (1998).

Multivariate data analysis (5th ed.). Upper Saddle River, NJ: Prentice Hall.

Hill, R. C.,

Griffiths, W. E., \&

Judge, G. G. (2003).

Econometria (2a ed.). São Paulo: Saraiva.

Hitt, M. A., Ireland, R. D., \&

Hoskisson, R. E. (2001).

Strategic management: competitiveness and globalization. Cincinnati, OH: South-Western College.
Jensen, M. C., \&

Meckling, W. (1976).

Theory of firm: managerial behavior, agency costs and ownership structure. Journal of Financial Economics, 3(4), 305-360.

Karier, T. (1993).

Beyond competition: the economics of mergers and monopoly power. Armonk, NY e Londres: M. E. Sharpe.

Khanna, T., \&

Palepu, K. (1998).

The future of business group in emerging countries: long run evidence from Chile. Boston, MA: Harvard Business School.

Khanna, T., \&

Rivkin, J. W. (2001).

Estimating the performance effects of business group in emerging markets. Strategic Management Journal, 22(1), 45-74.

Lang, L. H. P., \&

Stulz, R. M. (1994).

Tobin's q, corporate diversification and firm performance. Journal of Political Economy, 102(6), 1248-1281.

Lins, K., \&

Servaes, H. (2002).

Is corporate diversification beneficial in emerging markets? Financial Management, 31(2), 5-31.

Maddala, G. S. (2003). Introdução a econometria (3a ed.). Rio de Janeiro: LTC Editora. 
Markides, C. C., \&

Williamson, P. J. (1997).

Related diversification, core competences and corporate performance. In N. J. Foss (Ed.). Resources, firms and strategies: a reader in the resource-based perspective. Oxford: Oxford University Press.

Mendes-da-Silva, W. (2004).

Estratégia de diversificação: evidências de implicações para o desempenho financeiro de indústrias brasileiras. Anais do Congresso Latino-Americano de Estratégia, Itapema, SC, Brasil, 17.

Mendes-da-Silva, W., \&

Magalhães, P. A. O., Filho (2004, setembro).

Existe alguma associação entre governança corporativa e diversificação da firma? Um estudo empírico com indústrias brasileiras. Anais do Encontro Nacional da Associação Nacional de PósGraduação e Pesquisa em Administração, Atibaia, SP, Brasil, 27.

Moreira, J., \&

Planellas, M. (2003).

Diversification and performance: emerging vs. developed economies. Conference of the International Iberoamerican Academy of Management, São Paulo, SP, Brasil, 3.

Nachum, L. (1999).

Diversification strategies of developing firms. Journal of International Management, 5(2), 115-140.
Nachum, L. (2004).

Geographic and industrial diversification of developing country firms. Journal of Management Studies, 41(2), 275-294.

Parada, P., \&

Planellas, M. (2003).

Business diversification strategies followed by fast growth companies: a resource-based view approach to catalan multinational industrial companies. Conference of the International Iberoamerican Academy of Management, São Paulo, SP, Brasil, 3.

Pindyck, R., \&

Rubinfeld, D. (2004).

Econometria. Rio de Janeiro: Editora Campus.

Porter, M. E. (1998).

Da vantagem competitiva a estratégia corporativa. In C. A. Montgomery \& M. E. (Orgs.). Estratégia: a busca da vantagem competitiva (pp. 237-269). Rio de Janeiro: Campus.

Prahalad, C. K., \&

Hamel, G. (1990).

The core competence of the corporation. Harvard Business Review, 68(3), 79-91.

Servaes, H. (1996).

The value of diversification during the conglomerate merger wave. Journal of Finance, 51(4), 1201-1225. 
Singh, M.,

Mathur, I., \&

Gleason, K. C. (2004).

Governance and performance implications of diversification strategies: evidence from large U.S. firms. The Financial Review, 39(4), 489-526.
Stock, J. H., \&

Watson, M. W. (2004).

Econometria. São Paulo: Pearson Education. 